\title{
The diagnostic delay and treatment outcome of Clostridium difficile infection in the patients who underwent rectal surgery
}

\author{
Jaram Lee*, Seung-Seop Yeom*, Soo Young Lee, Chang Hyun Kim, Hyeong Rok Kim, Young Jin Kim \\ Department of Surgery, Chonnam National University Hwasun Hospital, Chonnam National University Medical School, Hwasun, Korea
}

Purpose: The bowel frequency of patients who had undergone rectal resection might be difficult to distinguish from the diarrhea of Clostridium difficile infection (CDI). The change of bowel movement following rectal surgery has been a challenge for the diagnosis of CDI and scarce studies discussed this diagnostic difficulty.

Methods: From January 2004 to January 2018, a total of 8,327 patients in a single tertiary colorectal cancer center was evaluated for CDI, and their medical records were ret rospectively reviewed. Bowel frequency and treatment outcomes were compared between the rectal resection group (RG) and colectomy group (CG). Diagnostic time was defined as the time interval between first diarrhea (more than three times a day) and pathologic confirmation date of $\mathrm{CDI}$.

Results: CDI incidence was 2.3\% (17/752) vs. $0.41 \%(31 / 7,575)$ between $R G$ and $C G(P<0.001)$. RG had frequent bowel movements than CG (RG: $13.56 \pm 6.16 /$ day vs. $C G: 8.39 \pm 6.23 /$ day; $P=0.010$ ), but the interval between the time of symptom and the time of CDI diagnosis was longer in the RG than in CG (RG: $1.38 \pm 3.34$ days vs. CG: $0.39 \pm 1.16$ days). A total of three mortalities has been occurred (RG: 2 vs. CG: 1), and the reasons were delayed diagnosis and omitted treatment.

Conclusion: Patients experienced significant bowel frequency after rectal surgery than after colectomy, and the delayed diagnosis was associated with mortality. Active surveillance for CDI should be performed for the patients who underwent rectal surgery to prevent morbidity and mortality from delayed diagnosis of $\mathrm{CDI}$, but sophisticated guideline also should be evaluated to reduce over-examinations.

Keywords: Clostridium difficile, Diagnosis, Colorectal surgery, Rectum, Colon

\footnotetext{
Received: Apr 19, 2019 Revised: Jun 20, 2019 Accepted: Jun 20, 2019 Correspondence to: Hyeong Rok Kim

Department of Surgery, Chonnam National University Hwasun Hospital, Chonnam National University Medical School, 322 Seoyang-ro, Hwasun 58128, Korea

Tel: +82-61-379-7646, Fax: +82-61-379-7661

E-mail: drkhr@jnu.ac.kr
}

ORCID: Jaram Lee (https://orcid.org/0000-0002-9660-0566), Seung-Seop Yeom (https://orcid.org/0000-0003-3651-222X), Soo Young Lee (https://orcid.org/00000002-9200-7933), Chang Hyun Kim (https://orcid.org/0000-0002-1954-189X), Hyeong Rok Kim (https://orcid.org/0000-0003-2737-0485), Young Jin Kim (https:// orcid.org/0000-0002-0104-8255)

*Jaram Lee and Seung-Seop Yeom contributed equally to this work.

Copyright (@) 2019 Korean Society of Surgical Oncology

This is an Open Access article distributed under the terms of the Creative Commons Attribution Non-Commercial License (http://creativecommons.org/licenses/by-nc/4.0) which permits unrestricted non-commercial use, distribution, and reproduction in any medium, provided the original work is properly cited.

\section{INTRODUCTION}

Clostridium difficile is an anaerobic gram-positive bacterium, and C. difficile infection (CDI) can result in severe colitis and death. Recently, the CDI incidence has been reported to be increasing, and it is now the most common hospital-acquired infection with significant effects on healthcare costs [1]. Previous studies reported that colorectal surgery was one of the risk factors for CDI, along with old age, gastric acid suppression, antibiotics use, patient, and hospital admission [2-5]. In especially, the patients who underwent rectal resection often undergo bowel frequency, and it may delay or make CDI diagnosis difficult. However, there are scarce studies about the risk for diagnostic delay of CDI in the patients who underwent rectal resection, except for some case reports [6,7]. So, the aim of this study was to compare the bowel frequency and treatment outcomes between CDI patients who underwent rectal can- 
cer surgery (resection group, RG) and who received colon surgery (colectomy group, CG).

\section{METHODS}

\section{Study design and variables}

From January 2004 to January 2018, medical records of a total of 8,327 patients who had undergone surgery in a single tertiary institution were retrospectively reviewed. All the patients in RG had diversion ileostomies, and the bowel frequencies were evaluated during the admission after an ileostomy repair operation.

Clinical data, preoperative antibiotic use, gastric acid suppression medication use, the number of bowel frequency, postoperative ileus (POI), treatment, and outcomes of patients were investigated. POI was defined as obstipation and intolerance of oral intake due to nonmechanical factors which occurred after surgery, and all the ileus was confirmed by abdominal X-ray or computed tomography (CT) scan. The time of bowel frequency and the CDI testing, diagnostic delay time, and the admission duration were also investigated, and we defined diagnostic delay time as the interval between "time of bowel frequency" and "time of the CDI test- ing." The admission duration was defined as the interval between "time of bowel frequency" and "time of discharge."

Two or three stool tests (toxin A/B array+polymerase chain reaction [PCR], toxin A/B array+stool culture, or toxin A/B array+P$\mathrm{CR}+$ stool culture) were performed simultaneously when patients had frequent bowel movements (more than three times a day) with or without leukocytosis, fever, and ileus, and the specimens were sent to the laboratory within an hour from the collection.

\section{Patient and public involvement}

Medical records of the patients who received surgical treatment for colorectal cancer (CRC) were retrospectively reviewed and analyzed. The positive results in the three stool tests for CDI are the enrollment criteria of the patients. This retrospective study is not harmful to the patients, and this study approved by the Institutional Review Board of Chonnam National University Hwasun Hospital (IRB No. CNUHH-2018-116) and was eligible for exemption of informed consent.

\section{Statistical analysis}

Categorical variables were compared by using Fisher exact test and

Table 1. Patient characteristics and treatment results

\begin{tabular}{|c|c|c|c|}
\hline Characteristic & Rectal resection $(n=17)$ & Colectomy $(n=31)$ & P-value \\
\hline Incidence rate (\%) & $2.3(17 / 752)$ & $0.41(31 / 7,575)$ & $<0.001$ \\
\hline Age (yr) & $65.00 \pm 9.16$ & $62.06 \pm 11.89$ & 0.393 \\
\hline Male sex & $11(68.8)$ & $19(61.3)$ & 0.753 \\
\hline $\operatorname{BMI}\left(\mathrm{kg} / \mathrm{m}^{2}\right)$ & $22.35 \pm 2.51$ & $22.55 \pm 3.47$ & 0.838 \\
\hline ASA & & & 0.881 \\
\hline Grade 3 & $2(11.8)$ & 7 (22.6) & \\
\hline Grade $1 \& 2$ & 15 (88.2) & $24(77.4)$ & \\
\hline Previous admission history & $5.69 \pm 4.60$ & $3.16 \pm 7.77$ & 0.239 \\
\hline Preoperative antibiotics use & $17(100)$ & $31(100)$ & NS \\
\hline H2 blocker use & $17(100)$ & $31(100)$ & NS \\
\hline Emergency operation & 0 & $6(19.4)$ & 0.082 \\
\hline Diversion enterostomy & 17 (100) & 0 & $<0.001$ \\
\hline Postoperative ileus & 0 & 7 (22.6) & 0.078 \\
\hline \multicolumn{4}{|l|}{ Combined symptoms } \\
\hline Fever & $4(23.5)$ & $12(38.7)$ & 0.272 \\
\hline Leukocytosis & $3(17.6)$ & $14(45.2)$ & 0.069 \\
\hline No. of bowel movements & $13.56 \pm 6.16$ & $8.39 \pm 6.23$ & 0.010 \\
\hline Time of bowel frequency $(P O D, A)$ & $6.56 \pm 4.15$ & $10.19 \pm 10.06$ & 0.175 \\
\hline Time of the CDI testing $(P O D, B)$ & $7.94 \pm 5.00$ & $10.58 \pm 10.16$ & 0.333 \\
\hline Diagnostic delay time $\left(B-A\right.$, day) ${ }^{a}$ & $1.38 \pm 3.34$ & $0.40 \pm 1.16$ & 0.154 \\
\hline Hospitalization period after symptom (day)a) & $6.38 \pm 3.63$ & $9.68 \pm 10.22$ & 0.175 \\
\hline Treatment for CDI (yes) & $13(76.4)$ & $21(67.7)$ & 0.062 \\
\hline Mortality & $2(11.8)$ & $1(3.2)$ & 0.264 \\
\hline
\end{tabular}

Values are presented as mean \pm standard deviation or number (\%).

BMI, body mass index; ASA, American Society of Anesthesiologists; NS, not significant; POD, postoperative days; CDI, Clostridium difficile infection.

a) Diagnostic delay time and the admission duration was defined as the interval between "time of bowel frequency ( $\geq 3$ times a day)-time of the CDI testing" and

"time of bowel frequency-time of discharge". 
linear by linear analysis. Continuous variables were compared by using the Mann-Whitney U-test or Kruskal-Wallis test. Results were considered significant at $\mathrm{P}<0.05$. Statistical analyses were conducted using SPSS software version 21.0 (IBM Corp., Armonk, NY, USA).

\section{RESULTS}

\section{Patients characteristics}

CDI incidence after RG and CG was 2.3\% (17/752) and 0.41\% $(31 / 7,575)(\mathrm{P}<0.001)$. Age, gender, body mass index were similar between two groups and, all patients received prophylactic antibiotics and postoperative gastric acid suppression treatments also. All patients in RG received low anterior resections with diversion ileostomies, and the reason of the ileostomy was due to anastomotic leakage $(2 / 17,11.8 \%)$ and for the anastomotic protection after preoperative chemoradiation for rectal cancers $(15 / 17,88.2 \%)$. There were no patients who received diversion enterostomy in the CG. Without statistical significance, RG had slightly lower American Society of Anesthesiologists (ASA) score and higher previous admission history $(5.69 \pm 4.60$ vs. $3.16 \pm 7.77)$ (Table 1$)$.
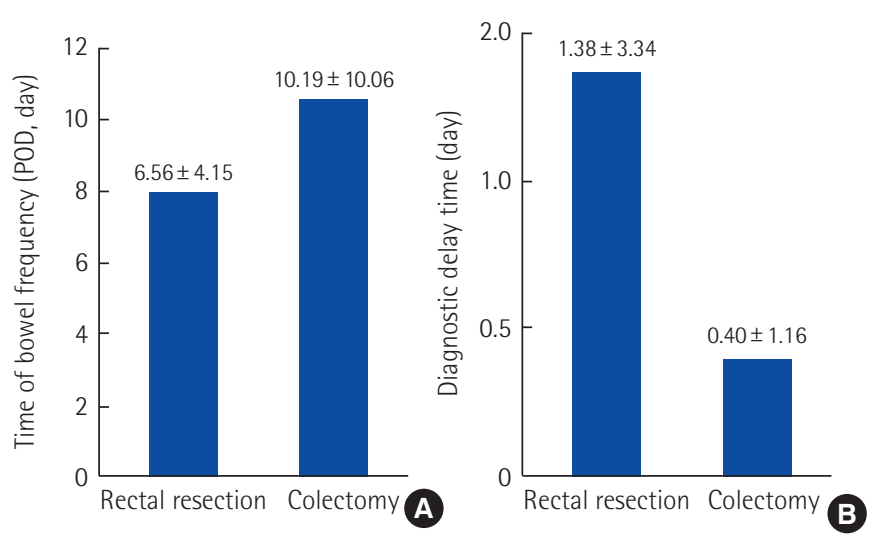

Fig. 1. Early symptom (A) and delayed diagnosis (B) of the rectal surgery group. POD, postoperative days.

\section{The bowel frequency and the diagnostic delay}

The incidence rate of CDI was higher in the RG (2.3\% vs. $0.41 \%$, $\mathrm{P}<0.001)$. The CDI patients' bowel frequency was detected in an earlier time in the RG than CG relatively (postoperative days [POD] $6.56 \pm 4.15$ vs. POD $10.19 \pm 10.06$ ), and CDI testings were also done in an earlier time in the RG (POD $7.94 \pm 5.00$ vs. POD $10.58 \pm 10.16)$. However, diagnostic times were relatively delayed in the RG than in the CG without statistical significance (1.38 \pm 3.34 days vs. $0.40 \pm 1.16$ days, $P=0.154$ ) (Table 1, Fig. 1 ).

\section{Treatment outcomes of CDI}

POIs were found in the seven patients (22.6\%) in CG, and there were no patients in RG with POI. The patients in CG experienced more frequent fever (23.5\% vs. $38.7 \%)$ and leukocytosis ( $17.6 \%$ vs. $45.2 \%)$, and longer admission duration ( $6.38 \pm 3.63$ days vs. $9.68 \pm$ 10.22 days) than the patients in RG, relatively. The $23.6 \%$ and $32.3 \%$ of patients in RG and CG omitted the treatments for CDI, two and one patients died from CDI (Table 1).

\section{Mortalities}

In the RG, two patients died from CDI (57-year-old male and 75-year-old female). CDI tests were performed at POD5 and $\mathrm{POD} 7$, but they did not receive any treatments for their CDI. The male patient died from the septic shock 2 days later from CDI testing. And the female patient discharged just after CDI testing before the positive pathologic results were reported because she had no complains and systemic symptom without bowel frequency. A few days later, she came back to the emergency department for the septic shock, and severe pancolitis was found at her CT scan. She admitted to the intensive-care unit but expired three days later due to multi-organ failures (Table 2).

In CG, a 78-year-old female patient experienced prolonged POI, and the leukocytosis and fever were combined with POI. Frequent bowel movements were identified at POD17, and the CDI test was promptly performed just after identifying the frequent bowel

Table 2. Mortality

\begin{tabular}{|c|c|c|c|c|c|c|c|c|c|}
\hline Age $(y r) / s e x$ & Surgery & BMI $\left(\mathrm{kg} / \mathrm{m}^{2}\right)$ & Comorbidity & $\begin{array}{l}\text { Previous } \\
\text { admission }\end{array}$ & $\begin{array}{l}\text { Postoperative } \\
\text { ileus }\end{array}$ & $\begin{array}{l}\text { Diagnosis } \\
\text { time (day) }\end{array}$ & Treatment & $\begin{array}{l}\text { Time of death } \\
\text { (day from } \\
\text { diagnosis) }\end{array}$ & CT finding \\
\hline $78 / F$ & $\begin{array}{c}\text { AR (emergent } \\
\text { operation) }\end{array}$ & 18.9 & $\begin{array}{l}\text { HTN, Parkinson } \\
\text { disease }\end{array}$ & 2 & 0 & P0D17 & IV vancomycin & 10 & Pancolitis \\
\hline $57 / M$ & LAR & 20.4 & - & 6 & $x$ & POD5 & Omitted & 2 & Pancolitis \\
\hline $75 / F$ & $L A R$ & 24.2 & - & 5 & $x$ & P0D7 & Omitted & 11 & $\begin{array}{l}\text { Ischemic } \\
\text { pancolitis }\end{array}$ \\
\hline
\end{tabular}

All patients expired from septic shock with multi-organ failure.

BMI, body mass index; CT, computed tomography; F, female; M, male; AR, anterior resection; HTN, hypertension; POD, postoperative days; IV, intravenous; LAR, low anterior resection. 
movement. And intravenous vancomycin was started as soon as possible, but the patient expired ten days later from the septic shock due to severe colitis.

\section{DISCUSSION}

$\mathrm{CDI}$ is a rare condition, and it looks that effective guidelines for CDI patients who received intestinal surgery are scarce. Especially, in patients who underwent rectal resection, their bowel frequency is difficult to distinguish with the symptoms of CDI. This study is a long-term result of a single tertiary CRC center, and we documented differences in the symptoms and diagnostic times, and treatment outcomes also between RG and CG. The patients in RG showed bowel frequency at earlier POD than that of CG, and the average number of bowel frequency was significantly higher than CG. About one day of the diagnostic delay in RG was identified compared to the CG $(1.38 \pm 3.34$ days vs. $0.40 \pm 1.16$ days $)$, and it seems to be associated with prolonged admission duration. And three mortalities were related to the delayed diagnosis and treatment omitting.

This study was performed with CRC patients, and the incidence rate of CDI was higher in the RG than CG. Few studies have examined the incidence rate of CDI in CRC patients. In recently, a team of researchers from Memorial Sloan Kettering Cancer Center and China investigated the pattern of $C$. difficile colonization in preoperative CRC patients [4], and the changes in the gut microbiota composition from the CRC would be associated with increased risk for CDI. But there are no comparative studies for the incidence rate between RG and CG, at least in our review. Recently review articles and a population-based study documented high incidence rate of CDI after ileostomy repair operation, and all patients in RG of this study received ileostomy repair operations. Although the high incidence rate of CDI in RG of this study could be affected by the ileostomy repair surgeries, but a caution for the CDI seems to be needed for the patients who underwent rectal surgery.

In this study, the symptom of CDI in CG was happened at a later time than RG, relatively. The reason for delayed symptom might be associated with $22.6 \%$ of POI patients in CG. POI is a common condition after abdominal surgery, and 14\%-68\% of the POI has been documented after colorectal surgery by previous randomized trials [8-10]. Ileus during the CDI treatment has been a well-known prognostic factor of CDI, and it was associated with leukocytosis, fever, postural hypotension and altered sensorium in previous studies $[11,12]$. However, there are no guidelines discussed the POI. Because of the small size of this study, we can not elucidate the exact effect of the POI, but our subanalysis suggests that POI would be related to the frequent systemic symptoms and longer treatment dura- tions (Supplemental Table S1). Discontinue of antibiotics use and repeated rectal swab test might be necessary to the patients who show POI with systemic symptoms like fever, significant leukocytosis, or unstable vital signs, but further studies should be performed.

In this study, many patients, a total of 14 patients (4 in RG vs. 10 in $\mathrm{CG}$ ) did not receive treatment for $\mathrm{CDI}$ after the pathologic diagnosis of CDI was confirmed by the toxin A/B assay or PCR or culture, interestingly, and most of the patients did not undergo CDI-associated morbidities. It is difficult to distinguish bacterial contamination and CDI, but two patients expired from septic shock due to severe colitis. Recent CDI treatment guidelines $[11,12]$ defines three times of unformed stool a day as a criterion for CDI diagnosis [13-16]. In this study, although RG showed about one day of a diagnostic delay than CG, most of CDIs could be diagnosed in early disease period, and the treatment outcomes were also favorable. However, the problem is that the number of bowel movements in most of the patients who had undergone rectal resection is more than three times a day $[14,17,18]$. Since that, for the CDI treatment, patients who had undergone rectal resection are inevitably exposed to the risk of over-examinations. More delicate diagnostic criteria should be investigated for patients who received the rectal surgery.

The change of bowel movement following rectal surgery has been a challenge for the diagnosis of CDI and scarce studies discussed this diagnostic difficulty. This is a unique study about CDI of the patients who received intestinal surgery. However, there are several limitations to this study. First, not all of the CDI-associated factors which have been documented related to the CDI incidence has been evaluated in this study. But the effects of bowel frequency and treatment outcome by CDI-associated with factors are not clear also. Second, the CDI incidence of this study may have been underestimated because there is the possibility of diagnostic omitting. Third, although we analyzed a large single center's database of the recent 15 years, the number of CDI patients was small because of the rare entity of CDI and many results failed to show statistical significances. Fourth, we performed tests for CDI when the number of bowel movements was more than three times a day, regardless of presence of CDI-related symptoms. Even if the test results were positive for CDI, many treatments were omitted according to doctors' opinions if they had no symptoms. Asymptomatic carriers are known to be capable of normal life without any treatments. So they may have affected the treatment outcome of our study. However, it is practically impossible to screen all patients to identify asymptomatic carriers. Although the small-size and retrospective design was the main weakness of this study, but a comparison of the bowel movement in patients who had CDI is barely possible by the prospective or population-based study. So we ex- 
pect that this study would make a contribution to the literature and will help many colorectal surgeons in making decision and care for CDI patients who received rectal cancer surgery.

In conclusion, this study showed the diagnostic delay of CDI in the patients who underwent rectal surgery, and mortalities were associated with delayed diagnosis and omitted treatment. In these patients who are expected to undergo frequent bowel movements, an active diagnostic test should be performed to prevent morbidity and mortality from the CDI if there are bowel movements more than three times, irrespective of whether they are accompanied by the additional systemic symptoms. However, it looks that the interest in the sophisticated guideline is also needed to reduce over-examination.

\section{CONFLICT OF INTEREST}

No potential conflict of interest relevant to this article was reported.

\section{ACKNOWLEDGMENTS}

SSY and JL performed the research, analyzed and interpreted the data, and revised the manuscript. HRK and YJK provided clinical expertise, assisted in data collection and compiling the database, and interpreted the data. SYL and CHK assisted in providing clinical expertise and data interpretation. SSY initiated the study, provided the study design, performed the statistical analyses, and wrote and extensively revised the manuscript. All authors have read and approved the final manuscript for publication.

\section{SUPPLEMENTARY MATERIALS}

Supplemental Table S1. Comparison of the patients with or without postoperative ileus

Supplemental data can be found at: https://doi.org/10.14216/ kjco. 19007

\section{REFERENCES}

1. Damle RN, Cherng NB, Flahive JM, Davids JS, Maykel JA, Sturrock PR, et al. Clostridium difficile infection after colorectal surgery: a rare but costly complication. J Gastrointest Surg 2014;18:1804-11.

2. Yeom CH, Cho MM, Baek SK, Bae OS. Risk factors for the development of Clostridium difficile-associated colitis after colorectal cancer surgery. J Korean Soc Coloproctol 2010;26:329-33.

3. Vonberg RP, Kuijper EJ, Wilcox MH, Barbut F, Tull P, Gastmeier P, et al. Infection control measures to limit the spread of Clostridium difficile. Clin Microbiol Infect 2008;14 Suppl 5:2-20.

4. Zheng Y, Luo Y, Lv Y, Huang C, Sheng Q, Zhao P, et al. Clostridium difficile colonization in preoperative colorectal cancer patients. Oncotarget 2017;8:11877-86.

5. Slimings C, Riley TV. Antibiotics and hospital-acquired Clostridium difficile infection: update of systematic review and meta-analysis. J Antimicrob Chemother 2014;69:881-91.

6. Abe I, Kawamura YJ, Sasaki J, Konishi F. Acute fulminant pseudomembranous colitis which developed after ileostomy closure and required emergent total colectomy: a case report. J Med Case Rep 2012;6:130.

7. Almerie MQ, Culverwell A, Mahon C. Clostridium difficile infection after ileostomy closure mimicking anastomotic leak. BMJ Case Rep 2015;2015:bcr2015210112.

8. van den Heijkant TC, Costes LM, van der Lee DG, Aerts B, Osinga-de Jong M, Rutten HR, et al. Randomized clinical trial of the effect of gum chewing on postoperative ileus and inflammation in colorectal surgery. Br J Surg 2015;102:202-11.

9. Lau C, Phillips E, Bresee C, Fleshner P. Early use of low residue diet is superior to clear liquid diet after elective colorectal surgery: a randomized controlled trial. Ann Surg 2014;260:641-7.

10. Boelens PG, Heesakkers FF, Luyer MD, van Barneveld KW, de Hingh IH, Nieuwenhuijzen GA, et al. Reduction of postoperative ileus by early enteral nutrition in patients undergoing major rectal surgery: prospective, randomized, controlled trial. Ann Surg 2014;259:649-55.

11. McDonald LC, Gerding DN, Johnson S, Bakken IS, Carroll KC, Coffin SE, et al. Clinical Practice Guidelines for Clostridium difficile Infection in Adults and Children: 2017 Update by the Infectious Diseases Society of America (IDSA) and Society for Healthcare Epidemiology of America (SHEA). Clin Infect Dis 2018;66: 987-94.

12. Steele SR, McCormick J, Melton GB, Paquette I, Rivadeneira DE, Stewart D, et al. Practice parameters for the management of Clostridium difficile infection. Dis Colon Rectum 2015;58:10-24.

13. van der Heijden JAG, Thomas G, Caers F, van Dijk WA, Slooter GD, Maaskant-Braat AJG. What you should know about the low anterior resection syndrome: clinical recommendations from a patient perspective. Eur J Surg Oncol 2018;44:1331-7.

14. Bryant CL, Lunniss PJ, Knowles CH, Thaha MA, Chan CL. Anterior resection syndrome. Lancet Oncol 2012;13:e403-8.

15. Pucciani F. A review on functional results of sphincter-saving surgery for rectal cancer: the anterior resection syndrome. Updates Surg 2013;65:257-63.

16. Ramage L, Qiu S, Kontovounisios C, Tekkis P, Rasheed S, Tan E. A systematic review of sacral nerve stimulation for low anterior resection syndrome. Colorectal Dis 2015;17:762-71.

17. Martellucci J. Low anterior resection syndrome: a treatment algorithm. Dis Colon Rectum 2016;59:79-82. 
18. Jimenez-Gomez LM, Espin-Basany E, Marti-Gallostra M, Sanchez-Garcia JL, Vallribera-Valls F, Armengol-Carrasco M. Low anterior resection syndrome: a survey of the members of the Ameri- can Society of Colon and Rectal Surgeons (ASCRS), the Spanish Association of Surgeons (AEC), and the Spanish Society of Coloproctology (AECP). Int J Colorectal Dis 2016;31:813-23. 
Supplemental Table S1. Comparison of the patients with or without postoperative ileus

\begin{tabular}{lccc}
\hline & Postoperative ileus $(+)(n=7)$ & Postoperative ileus $(-)(n=41)$ & P-value \\
\hline Age $(\mathrm{yr})$ & $62.86 \pm 14.51$ & $63.10 \pm 10.53$ & 0.958 \\
Male sex & $5(71.4)$ & $25(61.0)$ & 0.501 \\
BMI $\left(\mathrm{kg} / \mathrm{m}^{2}\right)$ & $20.16 \pm 2.34$ & $22.89 \pm 3.12$ & 0.033 \\
ASA grade 3 & $2(28.6)$ & $7(17.1)$ & 0.662 \\
Emergency operation & $1(14.3)$ & $5(12.5)$ & 0.643 \\
Previous admission history & $1.71 \pm 1.50$ & $4.43 \pm 7.41$ & 0.344 \\
Time to bowel frequency (POD, day) & $11.57 \pm 5.00$ & $8.50 \pm 9.10$ & 0.391 \\
Fever & $3(42.9)$ & $13(31.7)$ & 0.447 \\
Leukocytosis & $5(71.4)$ & $12(29.3)$ & 0.049 \\
Admission duration (POD, day) & $15.71 \pm 20.08$ & $7.62 \pm 3.88^{\mathrm{a})}$ & 0.328 \\
& & $8.04 \pm 3.92^{b)}$ & 0.078 \\
Treatment for CDI (yes) & $3(42.9)$ & $31(75.6)$ & 0.080 \\
Death & $1(14.3)$ & $2(4.9)$ & 0.391 \\
\hline
\end{tabular}

Values are presented as mean \pm standard deviation or number $(\%)$.

BMI, body mass index; ASA, American Society of Anesthesiologists; POD, postoperative days; CDI, Clostridium difficile infection.

a) Resection group+colectomy group. ${ }^{\text {b) }}$ Colectomy group only. 